\title{
Molecular testing and thyroid nodule management in North America
}

\author{
N. Paul Ohori \\ Department of Pathology, University of Pittsburgh Medical Center-Presbyterian, Pittsburgh, PA, USA \\ Correspondence to: N. Paul Ohori, MD. Department of Pathology, A610, UPMC-Presbyterian, 200 Lothrop Street, Pittsburgh, PA 15213, USA. \\ Email: ohorinp@upmc.edu.
}

\begin{abstract}
The incidence of thyroid cancer is rising for a variety of reasons. At the same time, the nomenclature revision of non-invasive encapsulated follicular-variant PTC to noninvasive follicular neoplasm with papillary-like nuclear features (NIFTP) has modified the incidence of thyroid cancer. Given that thyroid neoplasia is a molecular event, it is important for the thyroid physician to evaluate each patient systematically. Most thyroid cancers are sporadic; however, some are familial and may be associated with syndromes with genetic implications. Advances in radiologic imaging have made ultrasonography a near equivalent of gross examination. The American College of Radiology Thyroid Imaging, Reporting and Data System (ACR TI-RADS) classifies nodules from TR1 to TR5 and is valuable in determining which patients should be guided toward fine-needle aspiration (FNA) sampling. While FNA procedures and processing may be varied, the key elements are cytologic diagnosis and collection of samples for potential molecular testing. The Bethesda System for Reporting Thyroid Cytology (BSRTC) is commonly used and categorizes each FNA specimen into one of six diagnoses. The indeterminate diagnoses with risk of malignancy (ROM) ranging from 10-75\% comprise approximately 30\% of thyroid FNA cases and for these, molecular testing is beneficial. In North America, the two most common molecular platforms are Veracyte Afirma GSC and ThyroSeq v3. Both panels cover an extensive array of genomic alterations associated with thyroid neoplasia and a negative result from either test effectively refines the ROM of an Atypia of Undetermined Significance/ Follicular Lesion of Undetermined Significance (AUS/FLUS) or Follicular Neoplasm/Suspicious for a Follicular Neoplasm (FN/SFN) diagnosis to 3-4\%. Given that the refined ROMs are comparable to that of a Benign BSRTC diagnosis, these patients are recommended for observation of their nodules. However, differences exist in the implication of Afirma GSC-Suspicious and ThyroSeq v3-Positive molecular results with regard to the probability of cancer. For either test, the molecular test result should be integrated with other clinical parameters to determine if surgery is indicated and, if so, the extent of surgery.
\end{abstract}

Keywords: Thyroid; molecular testing; Afirma; ThyroSeq

Submitted May 02, 2020. Accepted for publication Jul 04, 2020.

doi: 10.21037/gs-2019-catp-26

View this article at: http://dx.doi.org/10.21037/gs-2019-catp-26

\section{Introduction}

The incidence of thyroid cancer has been increasing over the recent decades (Table 1) $(1,2)$. Much of the increase has been attributed to the increased detection of lowgrade, well differentiated neoplasms, in particular, differentiated papillary thyroid carcinoma (PTC). Medical and technological advances have contributed to the heightened clinical and radiologic sensitivity for small and subtle neoplasms, such as papillary thyroid microcarcinoma (PTMC). However, a large-scale study also observed an increase in the number of larger, more aggressive thyroid carcinomas with an increase in annual incidence-based mortality of approximately 1\% (3). The etiology for the 
Table 1 Factors involved in the changing incidence of thyroid cancer

\begin{tabular}{ll}
\hline Factors contributing to an increase in incidence & Factors contributing to a decrease in incidence \\
\hline $\begin{array}{l}\text { Heightened clinical sensitivity (including improved resolution of } \\
\text { radiologic imaging) }\end{array}$ & Decreased use of ionizing radiation therapy \\
$\begin{array}{l}\text { Environmental carcinogens } \\
\text { Concern for missing cancer }\end{array}$ & Nomenclature revision of noninvasive follicular-variant PTC to NIFTP \\
$\begin{array}{l}\text { Decreased threshold for diagnosing follicular-variant PTC by } \\
\text { pathologists }\end{array}$ & \\
\hline
\end{tabular}

increase is not known. Furthermore, there are biological changes with shifting proportions of encountered thyroid cancer subtypes. In the USA, molecular profiling of archival specimens of thyroid neoplasms from the last few decades has shown a decrease in the incidence of translocation-associated (e.g., RET/PTC) PTCs (which may be related to ionizing radiation) and a concurrent increase in RAS mutated PTCs, suggesting the involvement of environmental factors (4). Furthermore, comprehensive mapping of the genomic alterations in thyroid neoplasia has provided a clearer picture of the distinctions between nodular hyperplasia, low-grade neoplasms, and highgrade neoplasms (5-7). Nonetheless, the diagnosis of thyroid carcinoma is often in the eyes of the surgical pathologist and over the last three decades, an increase in the surgical pathology diagnosis of follicular-variant PTC was noted. However, the noninvasive forms of follicularvariant PTC were recognized to be extremely indolent and designated later as a non-malignant neoplasm. Recently, the 2016 nomenclature revision of non-invasive encapsulated follicular-variant PTC (categorically a cancer) to noninvasive follicular neoplasm with papillarylike nuclear features (NIFTP) (a non-malignant neoplasm but not entirely benign if left alone) resulted in decreases in the reporting of thyroid carcinomas and overtreatment of indolent neoplasms (8). However, a meta-analysis of reported series showed variability in the effect of the nomenclature revision with the prevalence of the NIFTP ranging from $<1 \%$ to $28 \%$ of all thyroid cancers (average $9.1 \%$ ) (9). Therefore, the impact of NIFTP on altering the incidence of thyroid cancer appears to depend on the geographic location, patient population, and the physicians involved in the care of these patients.

\section{Clinical evaluation}

Given the molecular events of thyroid neoplasia and the changing landscape described above, it is important for the thyroid physician to evaluate the patient with a thyroid nodule systematically to determine the risk of malignancy and the most appropriate management (Table 2). During the patient interview, clinical data regarding presenting features (e.g., compressive symptoms, dyspnea), prior biopsies or evaluations, radiation exposure, familial thyroid cancers and syndromes, autoimmune disorders, non-thyroid malignancies, etc. are recorded (10). History of radiation exposure is important for certain patients who may have received radiation therapy or resided in areas of nuclear disasters (e.g., Chernobyl, Fukushima). Inquiry into familial traits regarding thyroid cancer is important but extracting accurate information may be challenging if the patient and other family members are not aware of such syndromes. Over $90 \%$ of thyroid cancers are sporadic and due to somatic genomic alterations (11). The remaining familial cases are divided into familial non-medullary thyroid cancer (FNMTC) and familial medullary thyroid cancer (FMTC). FNMTC comprises approximately $60 \%$ of familial thyroid cancers and is divided further into nonsyndromic FNMTC $(\sim 95 \%)$ and syndromic FNMTC ( 5\%). The vast majority of FNMTC thyroid cancers is comprised of PTC ( $90 \%)$ and the remainder include follicular carcinoma, anaplastic carcinoma, and Hürthle cell carcinoma. The etiology for nonsyndromic FNMTC is not certain but may be related to involvement of susceptibility genes. Syndromic FNMTCs are associated with characteristic histologic subtypes, for example, familial adenomatosis polyposis syndrome (FAP)—cribriform morular variant PTC, PTENHamartoma (Cowden) syndrome-classic and follicularvariant PTC, Carney Complex Type I-PTC and follicular carcinoma, and DICER 1 syndrome-follicular carcinoma and follicular-variant PTC (10). FMTC which comprises approximately $40 \%$ of familial thyroid cancers is associated with mutations in the RET proto-oncogene on chromosome $10 \mathrm{q} 11.2$ and is subclassified further into multiple endocrine 
Table 2 Patient history, physical examination, laboratory findings, and radiologic imaging results and their implications (8)

\begin{tabular}{|c|c|}
\hline Clinical parameter & Implication \\
\hline Prior biopsies or evaluations & Prior biopsies and evaluations at outside institution may provide insight into the current situation \\
\hline Radiation exposure & Patients in areas of nuclear disaster are at risk of thyroid and other cancers \\
\hline \multirow{8}{*}{ Family history of thyroid cancer } & - Non-syndromic FNMTC ( 95\%) \\
\hline & Most are papillary thyroid carcinoma \\
\hline & - Syndromic FNMTC ( 5\%) \\
\hline & Familial adenomatosis polyposis syndrome \\
\hline & DICER 1 syndrome \\
\hline & Familial medullary thyroid cancer (FMTC) \\
\hline & - Multiple endocrine neoplasia 2A (95\%) \\
\hline & - Multiple endocrine neoplasia 2B (5\%) \\
\hline Thyroid function tests & Hyperthyroidism, autoimmune disorders \\
\hline Parathyroid hormone and serum calcium & Parathyroid disease \\
\hline
\end{tabular}

neoplasia (MEN) 2A (95\%) or 2B (5\%). For these patients, total thyroidectomy is recommended to remove all C-cells (with a germline RET mutation) and eliminate the risk of medullary thyroid carcinoma (MTC) development (12). Due to the serious implications for the patient and family, the decision to perform germline genetic testing should involve medical subspecialists and a genetic counselor (10).

Other aspects of the patient's clinical history that are important to obtain include rate of nodule or thyroid growth, history of autoimmune disease (e.g., thyroiditis, Graves disease), prior non-thyroid cancers, presence or absence of compressive symptoms (e.g., dysphagia, dyspnea), complaints suggesting thyroid hormone dysfunction (e.g., hyperthyroidism), prior head and neck surgeries and other risk factors (10). The physical examination not only focuses on the assessment of the thyroid nodule but also surveys signs and symptoms of thyroid hormone abnormalities and possible surgical risks. Additionally, voice assessment, laryngeal examination, and evaluation for the risk of recurrent laryngeal nerve injury are important aspects of pre-operative evaluation.

Clinical chemistry laboratory evaluation is also a part of routine evaluation. All patients should have their serum thyroid stimulating hormone (TSH) and possibly free T4 and total T3 levels tested to assess the possibility of hyperthyroidism (10). Serum calcium levels are taken routinely. If concern for parathyroid disease arises, measurement of parathyroid hormone (PTH) would be appropriate. While the use of routine serum calcitonin measurements is controversial, this test is indicated for patients at risk for MTC. Certainly, if the fine needle aspiration (FNA) test confirms or suspects MTC, serum calcitonin and carcinoembryonic antigen (CEA) measurements are indicated if they had not been performed beforehand. Germline RET proto-oncogene testing has serious implications for the patient and family and should involve counseling as mentioned above.

\section{Thyroid radiology}

Radiologic imaging is a critical component in thyroid nodule evaluation and often a key factor in deciding whether the patient will have an FNA (10). With advances made in recent decades, ultrasonography (US) has become highly 
Table 3 American College of Radiology Thyroid Imaging, Reporting and Data System (ACR TI-RADS) (11)

\begin{tabular}{ll}
\hline ACR TI-RADS designation & Risk of malignancy \\
\hline TR1 & $\leq 2 \%$ \\
TR2 & $\leq 2 \%$ \\
TR3 & $2.1-5.0 \%$ \\
TR4 & $5.1-20 \%$ \\
TR5 & $>20 \%$ \\
\hline
\end{tabular}

Table 4 Ultrasound-guided FNA parameters and details

\begin{tabular}{ll}
\hline Parameter & Specific details \\
\hline Needle gauge & Most commonly 25- or 27-gauge \\
Technique & Suction or capillary (non-aspiration) \\
& Direct smears \\
& $\bullet$ Alcohol-fixed Papanicolaou stain \\
& Liquid-based \\
& $\bullet$ ThinPrep \\
& $\bullet$ SurePath \\
Folecular testing & $\begin{array}{l}\text { Fresh specimen into a nucleic acid } \\
\text { preservative }\end{array}$ \\
collection methods & $\begin{array}{l}\text { Residual material collected for liquid- } \\
\text { based preparation }\end{array}$ \\
& $\begin{array}{l}\text { Cellular material scraped from direct } \\
\text { smears }\end{array}$ \\
Routine or requested by procedure & Performed by cytopathologist or \\
operator & cytotechnologist \\
\hline
\end{tabular}

sophisticated and may be viewed as in-situ gross examination of the thyroid gland. With widespread availability, US has become a routine aspect of patient evaluation. The entire thyroid gland (for nodule characteristics) and the central and lateral neck regions (for identification of abnormal lymph nodes) are examined. Organizations such as the American Thyroid Association (ATA) and the American College of Radiology (ACR) have developed guidelines to stratify the risk of malignancy (ROM). Of these two, the ACR Thyroid Imaging, Reporting and Data System (TI-RADS) has gained wide acceptance in the United States and incorporates the nodule composition, echogenicity, shape, margin, and echogenic foci as feature categories (13) (Table 3). Point scores based on radiologic findings are assigned to each feature category and the scores are tabulated for each case. Based on the total score, each case is given a designation from TR1 (benign) to TR5 (highly suspicious) with associated ROMs which range from $2 \%$ or less (TR1) to greater than $20 \%$ (TR5) (14). Subsequently, the decision to perform or not to perform an FNA is based on the size of the nodule and other factors. For TR3, TR4, and TR5 cases, nodules $\geq 2.5, \geq 1.5$, and $\geq 1.0 \mathrm{~cm}$ are recommended for FNA, respectively (15).

\section{Thyroid FNA technique and processing}

FNAs in North America are performed predominantly with ultrasound guidance (Table 4). For cases selected for FNA, a number of factors influence the process of thyroid nodule sampling. These include nodule size, location, ultrasound characteristics, and potential application of molecular testing. Nodules selected for FNA sampling are usually greater than $1 \mathrm{~cm}$. For nodules $\leq 1.0 \mathrm{~cm}$, FNAs are performed when the nodule is associated with metastatic disease or when there is suspicion for local invasion (16). Needle sizes most commonly used are 25 -gauge and 27 -gauge. However, larger caliber needles may be used under certain circumstances such as drainage of cyst contents. While the proceduralist's proficiency in FNA sampling has been shown to influence the yield of the diagnostic specimen, advantage of one particular technique (e.g., active suction or capillary technique) over another has not been established $(17,18)$. Nonetheless, individual proceduralists tend to prefer and utilize one method exclusively. Therefore, it is essential that proceduralists develop proficiency (minimizing unsatisfactory/nondiagnostic cases) regardless of the specific technique utilized. From each FNA pass, the specimen is expulsed for direct smear preparation (air-dried for Romanowskytype staining, alcohol-fixed for Papanicolaou staining), liquid-based preparation (collected in a specific fixative vial e.g., Cytolyt), or potential molecular testing (in a nucleic acid preservative vial). The number of passes dedicated to direct smears, liquid-based preparations (e.g., ThinPrep), and potential molecular testing varies from institution to institution. At our institution, a minimum of 2 direct smears for Papanicolaou staining, 2 direct smears for DiffQuik (Romanowsky-type) staining, and a ThinPrep slide (with Papanicolaou staining) are procured for cytology diagnosis and at least one pass or a portion of a pass is 
Table 5 BSRTC diagnoses, diagnostic features, and molecular testing guidelines (16)

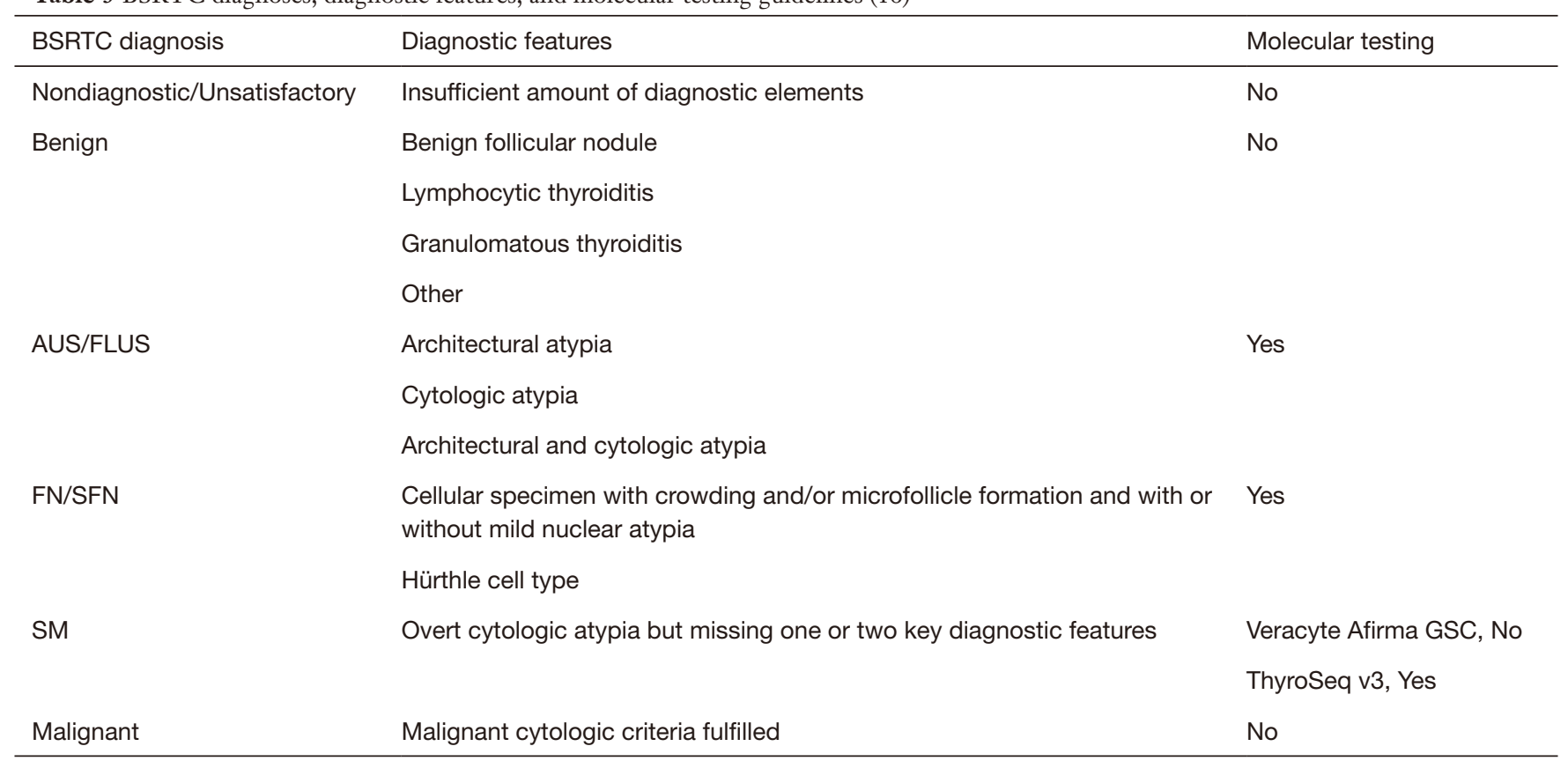

submitted for molecular testing. On-site evaluation may be ordered routinely or by request of the procedure operator and performed by the cytopathologist or cytotechnologist. While the slides for cytology diagnosis are processed routinely, the material collected for potential molecular testing is held until the cytology diagnosis is made. If the cytology diagnosis is indeterminate, consideration is given to performing molecular testing. However, patients are counseled on this matter and the choice of opting out of molecular testing is offered since some insurance policies may not cover the testing expenses adequately. From our experience, a large majority of patients opt for the testing. At some institutions, molecular testing is performed on residual cellular specimens in fixatives such as Cytolyt or by scraping cellular material from glass slides of direct smears. However, testing by these methods may be suboptimal as compared to using a dedicated specimen collected in nucleic acid preservative vials at the time of the FNA procedure. In addition to the routine processing mentioned above, cell blocks may be prepared in certain cases anticipating immunohistochemical staining (e.g., calcitonin for medullary carcinoma).

\section{Thyroid cytology reporting}

Most institutions reporting thyroid cytology in North
America use the Bethesda System for Reporting Thyroid Cytology (BSRTC) or a similar system (Table 5) (19). When compared to the first edition, the current second edition revised the management guidelines in accordance with the 2015 American Thyroid Association (ATA) guidelines and included molecular testing as an option for the management of indeterminate diagnoses (16). Furthermore, the second edition BSRTC acknowledged the influence of NIFTP on the risk of malignancy (ROM) estimation for each of the six diagnostic categories and provided two sets of ROM values (one set considering NIFTP as a "malignant" outcome and the other considering NIFTP as a "benign" outcome). Of these two sets, the ROM estimation with NIFTP as a "malignant" outcome with values that are comparable to those of the first edition BSRTC is used more commonly since NIFTP is considered a surgical disease. The management of the indeterminate BSRTC diagnosesAtypia of Undetermined Significance/Follicular Lesion of Undetermined Significance (AUS/FLUS), Follicular Neoplasm/Suspicious for a Follicular Neoplasm (FN/ SFN), Follicular Neoplasm, Hürthle Cell Type/Suspicious for a Follicular Neoplasm, Hürthle Cell Type (FNHCT/ SFNHCT) and Suspicious for Malignancy (SM) are most challenging since the ROMs fall in the intermediary range of $10 \%$ to $75 \%$.

In North American practices, most indeterminate cases 
Table 6 Comparison of Veracyte Afirma GSC and ThyroSeq v3

\begin{tabular}{lll}
\hline Parameter & Veracyte Afirma GSC & ThyroSeq v3 \\
\hline Molecular sample & Two FNA passes & One FNA pass \\
Diagnostic cytology interpretation & Central facility or local institution (if agreement made) & Central facility or local institution \\
Mutations analyzed & 346 genes (761 variants) & 112 genes (12,135 variants) \\
Gene fusions analyzed & 130 gene fusions & $>120$ gene fusions \\
$\begin{array}{l}\text { Chromosomal copy number } \\
\text { alterations }\end{array}$ & Loss of heterozygosity analysis & 10 chromosomal regions \\
Molecular results & Malignancy classifier positive & Negative (3\% cancer probability) \\
& GSC benign (4\% cancer probability) & Currently negative (5-10\% cancer probability) \\
& GSC suspicious ( 50\% cancer probability) & Positive-mutation details and cancer \\
& & probability stated for each
\end{tabular}

derive from one of the following reasons (19). Firstly, the FNA specimen may represent partial sampling of a neoplasm or lesion. With incomplete fulfillment of diagnostic criteria, a definitively benign or malignant diagnosis is not reached. Examples of such cases include focal manifestation of microfollicular structures that would not be sufficient for a FN/SFN diagnosis. Such cases are diagnosed often as AUS/FLUS with architectural atypia since the possibility of a hyperplastic nodule cannot be excluded. If the outcome is neoplastic, most are follicular-patterned neoplasms (e.g., follicular adenoma, NIFTP, follicular-variant PTC, follicular carcinoma) that do not show the robust nuclear features of PTC (e.g., nuclear elongation, numerous nuclear grooves and pseudoinclusions). In other instances, the nuclear features are concerning but present only focally. These cases are diagnosed as AUS/FLUS with cytologic atypia since the nuclear features overlap with those of benign neoplasms or lesions. Neoplastic outcomes for these cases are usually classic PTC, tall cell variant PTC and other PTCs with robust nuclear features of PTC. Secondly, the neoplasm or lesion may be adequately sampled by FNA with demonstration of a cellular specimen; however, the cytologic atypia is subtle or minimal. These cases are diagnosed most often as FN/SFN. On the hand, cases with overt cytologic atypia may have one or two key diagnostic features missing. These are diagnosed as SM. Thirdly, concerning non-epithelial or extracellular elements (e.g., psammoma bodies, thick colloid with multi-nucleated giant cells) may be identified in the absence of atypical epithelial cells. Such cases are categorized as AUS/FLUS with a description of the concerning elements. To further clarify the possible outcomes for the indeterminate diagnoses, molecular testing may be applied.

\section{Molecular testing}

The two most extensively studied molecular testing platforms are Veracyte Afirma and ThyroSeq (Table 6). The basic principles of the tests are to take a small sample of the FNA specimen and to run molecular tests on cases in which the cytology diagnosis is indeterminate. As mentioned above, multiple molecular specimen collection methods are available, although Veracyte Afirma and ThyroSeq each specify their recommended method. While the diagnostic cytology interpretation may be performed at the local institution or a centralized facility, molecular tests are performed exclusively at a centralized laboratory. The molecular test result, in conjunction with the cytology diagnosis, provides a refined ROM which aids in the management of the thyroid nodules.

Veracyte Afirma initially attracted attention with the landmark publication of its validation study (20). The current Veracyte Afirma Gene Sequencing Classifier (GSC) analyzes the expression pattern of more than 10,000 genes, of which 1,115 comprise the core genes (21). In addition, its Xpression Atlas analyzes 761 variants in 346 genes and 130 gene fusion pairs. Specialized collection test kits are provided for the user. In most instances, the FNA samples collected for diagnostic cytology are expelled into a Cytolyt vial for liquid-based ThinPrep processing and two additional dedicated FNA passes are placed into a vial of RNA preservative for potential molecular testing if indicated. Diagnostic cytology interpretation is performed usually at a centralized Veracyte facility unless an agreement 
for local interpretation is made. The Afirma GSC test is performed on AUS/FLUS and FN/SFN BSRTC diagnoses but is not recommended for SM cases. The RNA-Seq technology detects a variety of genetic alterations including point mutations, insertions/deletions, and gene fusions. Furthermore, Afirma GSC has built-in quality control measures for the detection of thyroid follicular epithelial cells and parathyroid cells. If the test is positive for one of its "malignancy classifiers" (e.g., BRAF, medullary carcinoma MTC, RET/PTC 1,3), further analysis with the main classifier is not performed. Otherwise, those cases tested by the main classifier are given the results of Afirma GSC benign (GSC-B) or Afirma GSC suspicious (GSC-S). Using a subset of cases from the original validation study, Afirma GSC achieved a sensitivity of $91 \%$, specificity of $68 \%$, negative predictive value (NPV) of $96 \%$, and positive predictive value (PPV) of $47 \%$ (21). According to Bayes' theorem, predictive values are dependent on disease prevalence. Therefore, the high negative predictive value (and thus the low ROM of $\sim 4 \%$ ), is based on the cancer prevalence of $\sim 10-40 \%$ for the AUS/FLUS and FN/SFN BSRTC categories. Furthermore, recent independent studies on Afirma GSC reported benign call rates of $66 \%$ to $76 \%$, which means that approximately two-thirds to three-quarters of the indeterminate cytology cases tested by Afirma GSC, have a de-escalated ROM of 4\% (22-25). For the remaining cases, the $\mathrm{ROM}$ is approximately $50 \%$. Therefore, for Afirma GSC-B cases, surveillance is considered, whereas for Afirma GSC-S and malignancy classifier positive cases, surgery is considered. The extent of surgery depends on additional factors such as clinical history (e.g., symptoms, thyroid function, comorbidities, familial history and possible syndromes), radiologic imaging features, specific genomic alteration (if stated), presence of co-existing thyroid disease, need for concurrent contralateral parathyroidectomy, surgical risk, and patient preference (10).

ThyroSeq takes a slightly different approach to molecular testing $(26,27)$. FNA samples collected for diagnostic cytology may be handled according to the protocol of the local institution (e.g., direct smears and/or liquid-based processing). The preferred specimen collection method for ThyroSeq molecular testing is to obtain at least one dedicated FNA pass which is placed into a nucleic acid preservative vial for potential molecular testing. Diagnostic cytology interpretation may be performed at the local institution or a centralized facility. ThyroSeq molecular tests are performed on all indeterminate BSRTC diagnoses
(AUS/FLUS, FN/SFN and SM). ThyroSeq originally started as a polymerase chain reaction and melting curve analysis test with a limited 7 -gene panel that had a high PPV but a low NPV $(28,29)$. Therefore, this test was regarded as a "rule-in" test originally. Over the last decade, ThyroSeq incorporated next generation sequencing (NGS) as the testing platform and evolved from ThyroSeq v1 (2013) to the current ThyroSeq v3 (2017) with an expanded 112gene panel covering single nucleotide variants, insertiondeletions, gene fusions, gene expression alterations, and copy number alterations. The ThyroSeq v3 validation study reported a sensitivity of $94 \%$, specificity of $82 \%$, NPV of $97 \%$, and PPV of $66 \%$ for the AUS/FLUS and FN/ SFN BSRTC categories (27). Like Veracyte Afirma GSC, ThyroSeq v3's high negative predictive value (and thus the low ROM of $\sim 3 \%$ ) is valid for cancer prevalence of $\sim 10$ $40 \%$ for AUS/FLUS and FN/SFN BSRTC categories. For the SM BSRTC diagnosis, a positive result may confirm the suspicion and guide the patient toward surgery (with total thyroidectomy in consideration, based on the genomic alteration and clinical features). However, a negative ThyroSeq v3 result, in the context of a SM diagnosis, still carries a $20 \% \mathrm{ROM}$ and therefore, patient counseling is advised for such cases.

The main difference between ThyroSeq $\mathrm{v} 3$ and Veracyte Afirma GSC is in the reporting of the positive molecular results (27). ThyroSeq v3's genomic classifier (GC) assigns 0,1 , or 2 points for each genomic alteration based its association with cancer (including NIFTP). The weighted sum is used to calculate the GC score, which in combination with the allelic frequency and type of genomic alteration is translated into the GC probability of cancer. This process was validated in a multicenter clinical trial which involved a large variety of thyroid (benign, NIFTP, malignant) and non-thyroid diseases. Since not all mutations are associated strongly with malignancies, very low-grade mutations such as PTEN are classified often as "currently negative" with probability of cancer of $\sim 5-10 \%$. The positive ThyroSeq v3 results have higher probabilities of cancer that are in the range of $\sim 30-99 \%$ (Table 7).

The detailed molecular results from ThyroSeq v3 contribute information toward determining the indication for surgery, the extent of surgery, and the prediction of outcomes (27). For negative ThyroSeq v3 results, usually observation is recommended and for ThyroSeq v3 "currently negative" cases, active surveillance is advised (with the exception of SM cytology cases). For the remaining positive cases, management depends on 
Table 7 ThyroSeq v3: common genomic alterations and probability of cancer or NIFTP (27)

\begin{tabular}{ll}
\hline Genomic Alterations & $\begin{array}{l}\text { Probability of cancer } \\
\text { or NIFTP }\end{array}$ \\
\hline PTEN & $\sim 5-10 \%$ \\
RAS alone & $40-80 \%$ \\
Gene expression alteration & $50-80 \%$ \\
THADA/IGF2 BP3 alone & $50-80 \%$ \\
Copy number alterations & $60-70 \%$ \\
RAS and other mutation(s) except TERT & $70-80 \%$ \\
PAX8/PPARG alone & $50-90 \%$ \\
TERT with or without other mutations & $80-95 \%$ \\
RET/PTC alone & $95 \%$ \\
BRAF V600E alone & $99 \%$ \\
Others & $30-95 \%$ \\
\hline
\end{tabular}

ThyroSeq v3's GC probability of cancer statement in conjunction with additional clinical parameters, including history (e.g., symptoms, thyroid function, comorbidities, familial history and possible syndromes), radiologic imaging features, presence of co-existing thyroid disease, need for concurrent contralateral parathyroidectomy, surgical risk, and patient preference. In this regard, a subset of patients with BRAF V600E mutation and the majority of patients with TERT promoter mutation (especially when identified with other mutations) are associated with aggressive thyroid cancer phenotypes. For these patients with high-risk mutations and a very high probability of cancer ( 99\%), total thyroidectomy may be advised if the other clinical indicators are consistent with such approach. Patients with RAS or RAS-like mutations (e.g., BRAF K601E, PAX8/ $P P A R g$ ) usually harbor follicular patterned neoplasms which commonly include follicular adenoma, NIFTP, follicularvariant PTC, and follicular carcinoma. When these mutations are identified alone, the probability of cancer or NIFTP is approximately $40-80 \%$. They usually correlate with an indolent follicular-patterned neoplasm for which thyroid lobectomy should be the initial consideration. Again, correlation with other clinical parameters are important to determine the extent of surgery.

Recently, the ThyroSeq v3 GC probability of cancer data have been shown to be potentially useful in calculating molecular-derived (MD) ROMs for the indeterminate diagnoses of individual institutions (30). When stating the ROM for each BSRTC diagnostic category on patient reports, individual institutions often reference the ROMs given in the current BSRTC text (19). However, the BSRTC ROMs are expressed in ranges, which are wide for the indeterminate diagnoses. (Table 5) Since practice patterns vary, the actual ROM for an indeterminate diagnosis (e.g., AUS/FLUS) may vary from institution to institution. Traditional cytologic-histologic calculations overestimate the ROMs when the denominator only consists of resection cases and underestimate the ROMs when the denominator consists of all cytology cases. Alternatively, MDROM can be calculated by pooling the ThyroSeq v3 GC probability of cancer data for each indeterminate category and determining its mean probability. This analysis is useful for AUS/FLUS and FN/SFN categories since only a subset of these cases undergo resection. With MDROM analysis, all cases with molecular testing (regardless of resection status) are included. Understanding one's own institutional MDROM for AUS/FLUS and FN/SFN diagnoses may enhance the ability of thyroid physicians to counsel patients.

Although thyroid molecular testing has been available for approximately 10 years, we are still in the early phases. Its overall success would depend on its impact on parameters such as the surgical resection rate, ratio of resected cancers (and NIFTPs) to benign nodules, patient survival and cost effectiveness when compared to more conservative approaches without the use of molecular testing. Furthermore, the approach to molecular testing in North America may not be applicable in other regions of the world with different ethnicities and cultures. For example, Asia has a relatively high prevalence of $B R A F$ V600E mutations $(\sim 80 \%)$ in thyroid cancers $(31,32)$. In this setting, testing for $B R A F$ V600E mutation alone may yield high sensitivity and specificity. However, in North America, there is a lower prevalence of $B R A F$ V600E mutations ( 40\%) (9). Therefore, utilization of molecular testing as effective rule-out tests with high negative predictive values requires a wider and more extensive panel of molecular markers in North America. Some institutions may take an intermediary approach of using a limited panel. For example, if a 7-gene panel is used, the specificity and PPV may be high since the panel often includes the most significant markers associated with thyroid cancer. However, the NPV is often less than $90 \%$ and would not be high enough for use as a "rule out" test $(33,34)$. More recently, a 10-gene panel (thyroid genetic classifier) offered as diagnostic kit, which may be utilized locally at the original institution, has been described (35). 
This type of testing takes a different approach from that of ThyroSeq v3 and Veracyte Afirma which require the original institution to submit the sample for molecular testing to centralized laboratories. Recent independent studies of international cohorts reported NPVs of $96 \%$ and $94 \%$, which would be acceptable as "rule out" tests (36). However, additional independent studies for the 10 gene panel (thyroid genetic classifier) with detailed performance characteristics and cost comparisons to the other molecular panels would be of interest. In lieu of molecular testing, some Asian thyroid practices have adopted a conservative active surveillance approach for the indeterminate (AUS/FLUS and FN/SFN) diagnoses and have achieved a malignancy outcome of $44 \%$ for these diagnoses (37). This figure is comparable to that achieved through ThyroSeq v2 and v3 molecular testing in North America (38). Conversely, whether conservative active surveillance without molecular testing can be accepted and achieve the same level of malignancy outcome in North America is uncertain since the patient cohorts and molecular characteristics are different between Asia and North America. Likewise, whether molecular testing is necessary, or for that matter, cost effective in Asia is also uncertain due to ethnic and cultural differences. Especially for patients in the USA, the true out-of-pocket cost for molecular testing is highly variable due to the wide range of insurance coverage. While the published cost of molecular testing may be a few thousand dollars, the true cost to the patient may be a few hundred dollars, if the patient has the "right" insurance (39). Unfortunately, data on the true out-of-pocket cost for molecular testing are not available publically. In our experience, the majority of patients in our system opt for testing when offered.

Management of thyroid nodules is multi-disciplinary such that the radiologist, cytopathologist and molecular pathologist convey the risk of the nodule, the clinician assimilates all of the patient's medical information and devises a plan, and the patient acknowledges, understands, accepts and participates in the management plan. In this regard, defining a universally acceptable hard border for the minimally acceptable lesion for resection (e.g., adenoma, NIFTP, carcinoma) is challenging to define, in part, due to cultural differences. Thyroid nodule management remains an art as well as a science.

\section{Acknowledgments}

Funding: None.

\section{Footnote}

Provenance and Peer Review: This article was commissioned by the Guest Editor (Kennichi Kakudo) for the series "Asian and Western Practice in Thyroid Pathology: Similarities and Differences" published in Gland Surgery. The article was sent for external peer review organized by the Guest Editor and the editorial office.

Conflicts of Interest: The author has completed the ICMJE uniform disclosure form (available at http://dx.doi. org/10.21037/gs-2019-catp-26). The series "Asian and Western Practice in Thyroid Pathology: Similarities and Differences" was commissioned by the editorial office without any funding or sponsorship. The author has no other conflicts of interest to declare.

Ethical Statement: The author is accountable for all aspects of the work in ensuring that questions related to the accuracy or integrity of any part of the work are appropriately investigated and resolved.

Open Access Statement: This is an Open Access article distributed in accordance with the Creative Commons Attribution-NonCommercial-NoDerivs 4.0 International License (CC BY-NC-ND 4.0), which permits the noncommercial replication and distribution of the article with the strict proviso that no changes or edits are made and the original work is properly cited (including links to both the formal publication through the relevant DOI and the license). See: https://creativecommons.org/licenses/by-nc-nd/4.0/.

\section{References}

1. Davies L, Welch HG. Increasing incidence of thyroid cancer in the United States, 1973-2002. JAMA 2006;295:2164-7.

2. Davies L, Welch HG. Current thyroid cancer trends in the United States. JAMA Otolaryngol Head Neck Surg 2014;140:317-22.

3. Lim H, Devesa SS, Sosa JA, et al. Trends in Thyroid Cancer Incidence and Mortality in the United States, 1974-2013. JAMA 2017;317:1338-48.

4. Jung CK, Little MP, Lubin JH, et al. The increase in thyroid cancer incidence during the last four decades is accompanied by a high frequency of BRAF mutations and a sharp increase in RAS mutations. J Clin Endocrinol Metab 2014;99:E276-85. 
5. Ye L, Zhou X, Huang F, et al. The genetic landscape of benign thyroid nodules revealed by whole exome and transcriptome sequencing. Nat Commun 2017;8:15533.

6. Xing M. Genetic-guided risk assessment and Management of Thyroid Cancer. Endocrinol Metab Clin North Am 2019;48:109-24.

7. Cancer Genome Atlas Research Network. Integrated genomic characterization of papillary thyroid carcinoma. Cell 2014;159:676-90.

8. Nikiforov YE, Seethala RR, Tallini G, et al. Nomenclature Revision for Encapsulated Follicular Variant of Papillary Thyroid Carcinoma: A Paradigm Shift to Reduce Overtreatment of Indolent Tumors. JAMA Oncol 2016;2:1023-9.

9. Bychkov A, Jung CK, Liu Z, et al. Noninvasive Follicular Thyroid Neoplasm with Papillary-Like Nuclear Features in Asian Practice: Perspectives for Surgical Pathology and Cytopathology. Endocr Pathol 2018;29:276-88.

10. Patel KN, Yip L, Lubitz CC, et al. The American Association of Endocrine Surgeons Guidelines for the Definitive Surgical Management of Thyroid Disease in Adults. Ann Surg 2020;271:e21-e93.

11. Xing M. Molecular pathogenesis and mechanisms of thyroid cancer. Nat Rev Cancer 2013;13:184-99.

12. Spanheimer PM, Ganly I, Chou J, et al. Long-Term Oncologic Outcomes After Curative Resection of Familial Medullary Thyroid Carcinoma. Ann Surg Oncol 2019;26:4423-9.

13. Tessler FN, Middleton WD, Grant EG, et al. ACR Thyroid Imaging, Reporting and Data System (TI-RADS): White Paper of the ACR TI-RADS Committee. J Am Coll Radiol 2017;14:587-95.

14. Tappouni RR, Itri JN, McQueen TS, et al. ACR TIRADS: Pitfalls, Solutions, and Future Directions. Radiographics 2019;39:2040-52.

15. Hoang JK, Middleton WD, Farjat AE, et al. Reduction in Thyroid Nodule Biopsies and Improved Accuracy with American College of Radiology Thyroid Imaging Reporting and Data System. Radiology 2018;287:185-93.

16. Haugen BR, Alexander EK, Bible KC, et al. 2015 American Thyroid Association management guidelines for adult patients with thyroid nodules and differentiated thyroid cancer: the American Thyroid Association guidelines task force on thyroid nodules and differentiated thyroid cancer. Thyroid 2016;26:1-133.

17. Maurya AK, Mehta A, Mani NS, et al. Comparison of aspiration vs non-aspiration techniques in fine-needle cytology of thyroid lesions. J Cytol 2010;27:51-4.
18. Schoedel KE, Tublin ME, Pealer K, et al. Ultrasoundguided biopsy of the thyroid: a comparison of technique with respect to diagnostic accuracy. Diagn Cytopathol 2008;36:787-9.

19. Ali SZ, Cibas ES. The Bethesda system for reporting thyroid cytopathology: definitions, criteria and explanatory notes, second edition. Cham, Switzerland: Springer, 2018.

20. Alexander EK, Kennedy GC, Baloch ZW, et al. Preoperative diagnosis of benign thyroid nodules with indeterminate cytology. N Engl J Med 2012;367:705-15.

21. Patel KN, Angell TE, Babiarz J, et al. Performance of a Genomic Sequencing Classifier for the Preoperative Diagnosis of Cytologically Indeterminate Thyroid Nodules. JAMA Surg 2018;153:817-24.

22. Angell TE, Heller HT, Cibas ES, et al. Independent Comparison of the Afirma Genomic Sequencing Classifier and Gene Expression Classifier for Cytologically Indeterminate Thyroid Nodules. Thyroid 2019;29:650-6.

23. Endo M, Nabhan F, Porter K, et al. Afirma Gene Sequencing Classifier Compared with Gene Expression Classifier in Indeterminate Thyroid Nodules. Thyroid 2019;29:1115-24.

24. Wei S, Veloski C, Sharda P, et al. Performance of the Afirma genomic sequencing classifier versus gene expression classifier: An institutional experience. Cancer Cytopathol 2019;127:720-4.

25. San Martin VT, Lawrence L, Bena J, et al. Real-world Comparison of Afirma GEC and GSC for the Assessment of Cytologically Indeterminate Thyroid Nodules. J Clin Endocrinol Metab 2020;105:dgz009.

26. Nikiforova MN, Mercurio S, Wald AI, et al. Analytical performance of the ThyroSeq 33 genomic classifier for cancer diagnosis in thyroid nodules. Cancer 2018;124:1682-90.

27. Steward DL, Carty SE, Sippel RS, et al. Performance of a multigene genomic classifier in thyroid nodules with indeterminate cytology: a prospective blinded multicenter study. JAMA Oncol 2019;5:204-12.

28. Nikiforov YE, Steward DL, Robinson-Smith TM, et al. Molecular testing for mutations in improving the fineneedle aspiration diagnosis of thyroid nodules. J Clin Endocrinol Metab 2009;94:2092-8.

29. Ohori NP, Nikiforova MN, Schoedel KE, et al. Contribution of molecular testing to thyroid fine-needle aspiration cytology of "follicular lesion of undetermined significance/atypia of undetermined significance". Cancer Cytopathol 2010;118:17-23.

30. Ohori NP, Landau MS, Manroa P, et al. Molecular-derived 
estimation of risk of malignancy for indeterminate thyroid cytology diagnoses. J Am Soc Cytopathol 2020. [Epub ahead of print].

31. Lee SE, Hwang TS, Choi YL, et al. Molecular Profiling of Papillary Thyroid Carcinoma in Korea with a High Prevalence of BRAFV600E Mutation. Thyroid 2017;27:802-10.

32. Kim H, Kim BH, Kim YK, et al. Prevalence of BRAFV600E Mutation in Follicular Variant of Papillary Thyroid Carcinoma and Non-Invasive Follicular Tumor with Papillary-Like Nuclear Features (NIFTP) in a BRAFV600E Prevalent Area. J Korean Med Sci 2018;33:e75.

33. Poller DN, Glaysher S. Molecular pathology and thyroid FNA. Cytopathology 2017;28:475-81.

34. Eszlinger M, Böhme K, Ullmann M, et al. Evaluation of a Two-Year Routine Application of Molecular Testing of Thyroid Fine-Needle Aspirations Using a Seven-Gene Panel in a Primary Referral Setting in Germany. Thyroid 2017;27:402-11.

35. González HE, Martínez JR, Vargas-Salas S, et al. A 10-

Cite this article as: Ohori NP. Molecular testing and thyroid nodule management in North America. Gland Surg 2020;9(5):1628-1638. doi: 10.21037/gs-2019-catp-26
Gene Classifier for Indeterminate Thyroid Nodules: Development and Multicenter Accuracy Study. Thyroid 2017;27:1058-67.

36. Zafereo M, McIver B , Vargas-Salas S, et al. A Thyroid Genetic Classifier Correctly Predicts Benign Nodules With Indeterminate Cytology: Two Independent, Multicenter, Prospective Validation Trials. Thyroid 2020;30:704-12.

37. Kakudo K, Higuchi M, Hirokawa M, et al. Thyroid FNA cytology in Asian practice-Active surveillance for indeterminate thyroid nodules reduces overtreatment of thyroid carcinomas. Cytopathology 2017;28:455-66.

38. Jug R, Foo WC, Jones C, et al. High-risk and intermediatehigh-risk results from the ThyroSeq v2 and $\mathrm{v} 3$ thyroid genomic classifier are associated with neoplasia: Independent performance assessment at an academic institution. Cancer Cytopathol 2020. [Epub ahead of print].

39. Nishino M, Nikiforova M. Update on Molecular Testing for Cytologically Indeterminate Thyroid Nodules. Arch Pathol Lab Med 2018;142:446-57. 